\title{
Overexpression of Heat Shock Factor 1 Masks the Heavy Metal Response of the Heat Shock Protein 70 (hsp70) Gene Promoter
}

\author{
Rie Uenishi, Kaoru Suzuki, and Shinji Koizumi* \\ Department of Health Effects Research, National Institute of Industrial Health, 6-21-1, Nagao, Tama-ku, Kawasaki 214-8585, Japan
}

(Received November 29, 2004; Accepted December 3, 2004; Published online December 13, 2004)

\begin{abstract}
Transcription of the heat shock protein 70 (hsp70) gene is induced by heavy metals including $\mathrm{Cd}$ and $\mathrm{Zn}$, probably through the interaction between the heat shock elements (HSE) and heat shock factor 1 (HSF1) binding to it. We studied the mechanism of this transcriptional activation by means of electrophoretic mobility shift assay (EMSA) and transient transfection assay. In HeLa cells, the DNA-binding activity of HSF1 was activated by $Z n$ in a concentration-dependent manner similar to that for $h s p 70$ gene expression, suggesting the direct contribution of HSF1 to the metalinduced transcriptional activation. To facilitate the analysis of the metal-regulatory mechanism, we attempted to establish a system that can reproduce the metal activation of overexpressed recombinant HSF1. In cells transfected with an HSF1 expression vector, strong HSE-binding activity was detected in EMSA, and reporter gene expression driven by the $h s p 70$ gene promoter was markedly increased. However, no $\mathrm{Zn}$ response was observed in either assay. The overexpressed HSF1 appeared to cause the constitutive activation of reporter expression, which is an intriguing feature that might reflect an aspect of the hsp70 gene regulation.
\end{abstract}

Key words — zinc, heat shock factor 1 , heat shock protein 70 gene, heat shock element, metal-responsive element-binding transcription factor-1, metal-responsive element

\section{INTRODUCTION}

It is known that many mammalian genes are transcriptionally activated by heavy metals. ${ }^{1,2)}$ However, studies on the metal-inducible genes have almost

\footnotetext{
*To whom correspondence should be addressed: Department of Health Effects Research, National Institute of Industrial Health, 6-21-1, Nagao, Tama-ku, Kawasaki 214-8585, Japan. Tel.: +81-44-865-6111; Fax: +81-44-865-6124; E-mail: koizumi @niih.go.jp
}

been restricted to those coding for metallothioneins (MTs), a family of low molecular weight metal-binding proteins. The $M T$ genes are activated by various heavy metals such as $\mathrm{Cd}, \mathrm{Zn}, \mathrm{Cu}$ and $\mathrm{Hg} .{ }^{3}$ Metal response is mediated by a cis-regulatory element, metal-responsive element (MRE), and the MREbinding transcription factor-1 (MTF-1) plays the major role in this process. ${ }^{4}$ By contrast, little is known for other metal-inducible genes.

The heat shock protein 70 ( $h s p 70)$ gene coding for the 70-kdalton heat shock protein, a heat-inducible gene, is also known to be activated by heavy metals. ${ }^{5)}$ With regard to this gene, the heat shock element (HSE) and heat shock transcription factor 1 (HSF1) binding to it were assumed to be involved in the metal regulation. ${ }^{6,7)}$ No cross-recognition of the target sequences was observed between MTF-1 and HSF1, ${ }^{7)}$ suggesting that HSF1 mediates metal response through a pathway distinct from the MTF1/MRE system. In the present study, we approached the mechanism of the heavy metal-induced activation of $h s p 70$ by means of electrophoretic mobility shift assay (EMSA) and transient transcription assay.

\section{MATERIALS AND METHODS}

Cell Culture, Transfection and the Preparation of whole Cell Extracts — HeLa S3 cells (CCL 2.2) were grown in Eagle's minimum essential medium supplemented with $10 \%$ calf serum and nonessential amino acids at $37^{\circ} \mathrm{C}$ in $5 \% \mathrm{CO}_{2}$. For the preparation of whole cell extracts (WCEs), cells were plated at $1 \times 10^{6}$ cells/10-cm Falcon plastic dish in $10 \mathrm{ml}$ of the medium with $100 \mu \mathrm{g} / \mathrm{ml}$ of streptomycin and $100 \mathrm{units} / \mathrm{ml}$ of penicillin. After an overnight incubation, the cells were transfected with $5 \mu \mathrm{g}$ of the HSF1 expression vector pCI-HSF1 or a control vector by a standard calcium phosphate method. For the latter, pUC19 or the empty vector pCI (described 
below) was used. The molar concentration of $\mathrm{pCI}$ was adjusted to be identical with that of pCI-HSF1, and pUC19 was added to make the total DNA input $5 \mu \mathrm{g}$. After a 46-hr incubation, the cells were cultured with or without $\mathrm{ZnSO}_{4}$ for $6 \mathrm{hr}$ further. WCEs were prepared as previously described. ${ }^{7}$

Western Blot Analysis — Western blot analysis was performed as previously described. ${ }^{8)}$ To detect HSF1, a rabbit anti-human HSF1-IgG and a goat anti-rabbit IgG-IgG/peroxidase conjugate (Santa Cruz Biotechnology, Inc., CA, U.S.A.) were used as the primary and secondary antibodies, respectively.

EMSA and Supershift Assay —_ EMSA was performed as previously described. ${ }^{7)}$ Synthetic 20-bp double-stranded oligonucleotides containing the MREa of the human MT-llA gene and the HSE of the human $h s p 70$ gene $^{7)}$ were end-labeled with $[\gamma-$ ${ }^{32} \mathrm{P}$ ] ATP (ICN Biomedicals, Inc., CA, U.S.A.) and T4 polynucleotide kinase (Takara Bio, Inc., Shiga, Japan), and used as probes. For supershift assay, a rabbit IgG directed to human HSF1 (Santa Cruz Biotechnology, Inc. or Affinity BioReagents, Inc., CO, U.S.A.) or HSF2 (Santa Cruz Biotechnology, Inc.) was added to the EMSA reaction mixture after binding reaction, and incubated at $25^{\circ} \mathrm{C}$ for $20 \mathrm{~min}$ further before electrophoresis.

Plasmid Construction — A plasmid containing the $h s p 70$ gene promoter ( $\mathrm{pH} 2.8)$ was a generous gift from Dr. R. I. Morimoto. $\left.{ }^{9}\right)$ The NcoI/BamHI fragment containing the $h s p 70$ promoter (NcoI site had been blunt-ended; spanning from -270 to +156 ) was cloned between the SmaI and BamHI sites within the multi-cloning site of pUC19 to generate pHSP70pr. A unique KpnI site was located just 5' of the SmaI site of pHSP70pr.

The hsp70 promoter has an HSE between -105

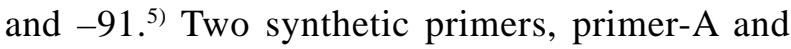
primer-B were used in the following steps. PrimerA has a sequence $5^{\prime}$-TGGGTACCAACCCCTGGA ATATTCCCGACC-3', containing an N-terminal KpnI site in addition to the HSE and flanking sequences. Primer-B has a sequence $5^{\prime}$-TCCTCAGG CTAGCCGTTATCCG-3', containing the NheI site and flanking sequences that are located just downstream from the transcription start site of the $h s p 70$ gene. The sequence between the two primers was amplified by PCR with pHSP70pr as a template. The PCR product was digested with KpnI and NheI, and the KpnI/NheI fragment of the original pHSP70pr was replaced with this truncated fragment generating $\mathrm{p} \Delta(-112)$. The KpnI/BamHI fragments from
pHSP70pr and $\mathrm{p} \Delta(-112)$ were cloned between the KpnI and BglII sites in the multi-cloning site of pCAT3 Basic (Promega, WI, U.S.A.) to place the chloramphenicol acetyltransferase (CAT) reporter gene under the control of the $h s p 70$ promoter or its deletion mutant, generating $\mathrm{pHSP70prCAT}$ and $\mathrm{p} \Delta(-$ 112)CAT, respectively.

A plasmid containing the human HSF1 cDNA sequence was a generous gift from Dr. C. Wu. ${ }^{10)}$ An EcoRI fragment containing the entire HSF1 coding region was excised from the plasmid, and cloned into the EcoRI site of an expression vector pCI with the cytomegalovirus (CMV) promoter (Promega) to generate pCI-HSF1.

Transient Transfection Assay — HeLa S3 cells were plated at $4 \times 10^{5}$ cells $/ 6$-cm plastic dish and incubated overnight. The cells were transfected with $3.2 \mu \mathrm{g}$ of the reporter vector pHSP70prCAT or an equimolar amount of $\mathrm{p} \Delta(-112) \mathrm{CAT}$ (in this case, pUC19 was added to make the total DNA amount $3.2 \mu \mathrm{g}$ ) by a standard calcium phosphate procedure. As a reference vector, $0.8 \mu \mathrm{g}$ of pRSVL that expresses the firefly luciferase ${ }^{11)}$ was co-transfected. In experiments with HSF1 overexpression, $0.4 \mu \mathrm{g}$ of pCI-HSF1 (or the corresponding empty vector pCI as a control) was co-transfected. After $46 \mathrm{hr}, \mathrm{ZnSO}_{4}$ was added to the medium, and the cells were incubated for $6 \mathrm{hr}$ further. The levels of CAT and luciferase in the cell extracts were determined by enzyme-linked immunosorbent assay (ELISA) and enzyme activity measurement, respectively. ${ }^{11)} \mathrm{Re}$ porter activity was calculated by normalizing the CAT protein amount to the unit luciferase activity.

\section{RESULTS}

We previously reported that HSE-binding activities were induced in Zn-treated HeLa cells. ${ }^{7)}$ It has been confirmed by a supershift analysis that these complexes contain HSF1 (S. Koizumi, unpublished results). To examine the dose-dependent effect of $\mathrm{Zn}$, WCEs were prepared from HeLa cells treated with different concentrations of $\mathrm{Zn}$, and the HSF1/ HSE complexes were detected by EMSA (Fig. 1). Two retarded bands were observed in the cells treated with $200 \mu \mathrm{M}$ of $\mathrm{ZnSO}_{4}$ for $6 \mathrm{hr}$ (lane 11), but not in untreated cells (lane 7) and the cells treated with $100 \mu \mathrm{M} \mathrm{ZnSO}_{4}$ (lanes 9). The $\mathrm{Zn}$ dependency of the HSF1/HSE complexes observed in this experiment is closely related to that of $h s p 70$ mRNA expression. ${ }^{7)}$ However, activation did not occur by the ad- 


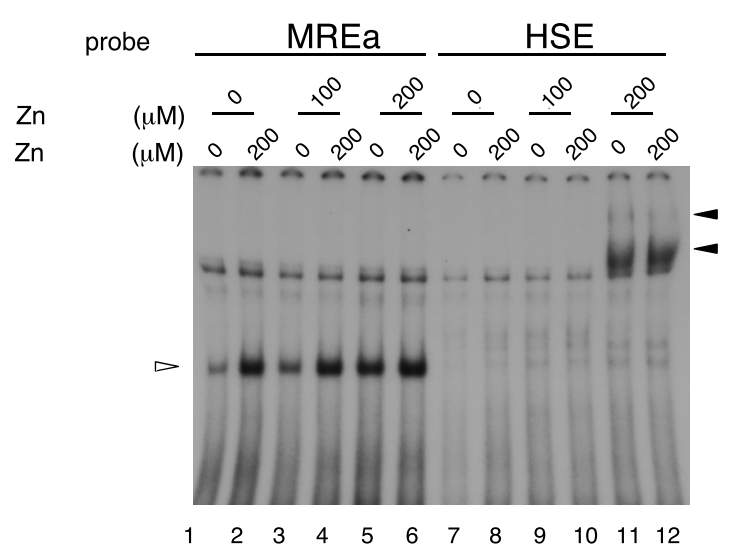

Fig. 1. Induction of HSE-Binding Activities in a Manner Dependent on Zn Concentration

HeLa cells were incubated with $0 \mu \mathrm{M}$ (lanes 1, 2, 7 and 8), $100 \mu \mathrm{M}$ (lanes 3, 4, 9 and 10), or $200 \mu \mathrm{M}$ (lanes 5, 6, 11 and 12) of ZnSO for 6 hr. WCEs were prepared from the cultures, and analyzed for MRE- (lanes 1 to 6 ) or HSE-binding activity (lanes 7 to 12) by EMSA. Binding reactions were carried out in the absence (lanes 1, 3, 5, 7, 9 and 11) or presence (lanes 2, 4, 6, 8, 10 and 12) of $200 \mu \mathrm{M} \mathrm{ZnSO}_{4}$. The white and black arrowheads indicate the bands retarded by MTF-1 and HSF1, respectively.

dition of Zn in vitro. The HSF1 complexes were not induced by the addition of $200 \mu \mathrm{M} \mathrm{Zn}$, which is sufficient for induction in vivo, to the protein-DNA binding reaction (Fig. 1, lanes 8 and 10). Furthermore, $\mathrm{Zn}$ added in vitro did not increase the level of the HSF1 complexes induced by $\mathrm{Zn}$ added in vivo (lane 12; compare with control in lane 11).

As a reference, we also examined the effect of $\mathrm{Zn}$ on the DNA-binding of another transcription factor, MTF-1. The binding of MTF-1 to its cognate recognition sequence MRE was activated by $\mathrm{Zn}$ at $100 \mu \mathrm{M}$, and more strongly at $200 \mu \mathrm{M}$ (Fig. 1, lanes 3 and 5, respectively; compare with untreated control in lane 1). This Zn dependency of MTF-1 also correlated well to that of MT mRNA expression. ${ }^{7)}$ In contrast to the HSF1 activity, however, induction of the MRE-binding activity was reproduced by the addition of $\mathrm{Zn}$ to the protein-DNA binding reaction (lanes 2 and 4; compare with controls in lanes 1 and 3 , respectively). Even with the WCE from the cells not exposed to Zn, which showed only a weak DNAbinding activity (lane 1), MTF-1 was fully activated by $200 \mu \mathrm{M} \mathrm{Zn}$ added in vitro (Fig. 1, lane 2; compare with the cells treated with $200 \mu \mathrm{M} \mathrm{Zn}$ in vivo in lane 5). Thus, these two transcription factors are apparently different in the response to $\mathrm{Zn}$.

To approach the mechanism of $\mathrm{Zn}$ response through HSF1/HSE further, we tried to establish a transient transfection system with overexpressed recombinant human HSF1. A plasmid pCI-HSF1, that contains the human HSF1 cDNA driven by the CMV promoter, was introduced into HeLa cells. The expression of the recombinant protein was monitored by western blot analysis (Fig. 2a). A high level of expression of a protein identical in mobility with the endogenous HSF1 (75 to $85 \mathrm{kDa}^{12)}$ ) was observed in WCE from the cells transfected with pCI-HSF1 (lane 2). We then analyzed the WCE by EMSA (Fig. 2b). Strong expression of the HSE-binding activities was observed in these cells (lane 7; compare with control in lane 5 without overexpression), but $\mathrm{Zn}$ treatment in vivo did not increase their levels further (lane 8; compare with lane 7). Surprisingly, the induction of endogenous HSF1 by Zn, as observed in Fig. 1, was abolished in the cells transfected with the empty vector pCI (lane 6). Such an effect was not observed for MTF-1 (lane 2). In addition, the overexpression of HSF1 did not affect the DNA-binding activity of MTF-1 (compare lanes 2 and 4). It was further confirmed if the HSEbinding activities actually represent HSF1 by supershift analysis (Fig. 2c). The HSE complexes in cells overexpressing HSF1 (lane 1) were supershifted by two different anti-HSF1 antibody products (lanes 2 and 3), but not by an anti-HSF2 antibody (lane 4). These results demonstrated that the observed HSE-binding activities were derived from the overexpressed recombinant HSF1.

To estimate the transcriptional activity of the endogenous HSF1 and the overexpressed HSF1, transient transfection assays were carried out (Fig. 3). Two reporter plasmids were constructed: pHSP70prCAT that carries the 5 '-flanking region of the $h s p 70$ gene $(-270$ to +156$)$ linked to the CAT reporter gene and $\mathrm{p} \Delta(-112) \mathrm{CAT}$ that lacks the distal part of the $h s p 70$ promoter $(-270$ to -113$)$ but re- 
(a)

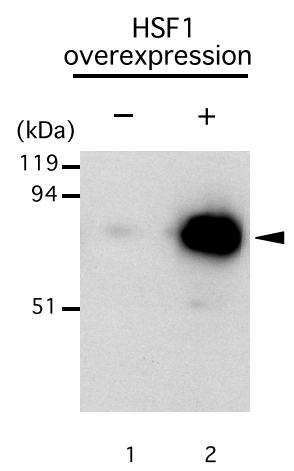

(b)

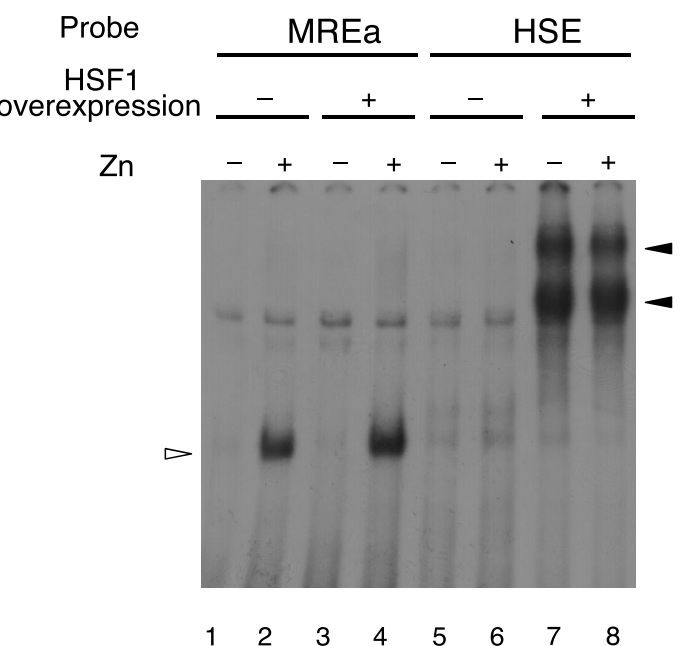

(c)

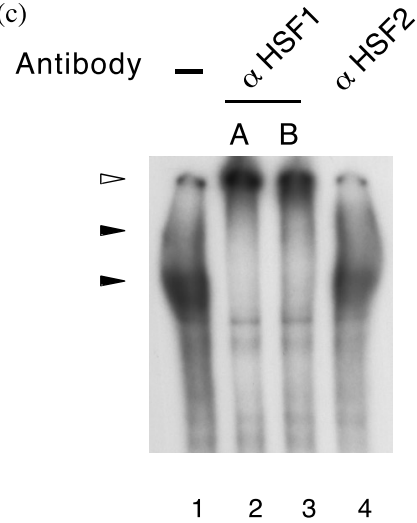

Fig. 2. Overexpression of HSF1 in HeLa Cells

a, Western blot analysis. HeLa cells were transfected with pUC19 (lane 1) or pCI-HSF1 (lane 2), incubated for $46 \mathrm{hr}$ and exposed to $200 \mu \mathrm{M} \mathrm{ZnSO}_{4}$ for $6 \mathrm{hr}$. WCEs ( $7 \mu \mathrm{g}$ protein/lane) were electrophoresed in SDS-7.5\% polyacrylamide gel, and HSF1 was detected by immunoblotting with an anti-HSF1 antibody. Positions of the marker proteins are indicated on the left: $\beta$-galactosidase (119 kDa), bovine serum albumin (94 kDa) and ovalbumin (51 kDa). b, EMSA. HeLa cells were transfected with pCI (lanes 1, 2, 5 and 6) or pCI-HSF1 (lanes 3, 4, 7 and 8), incubated for $46 \mathrm{hr}$, and exposed to $200 \mu \mathrm{M} \mathrm{Zn}$ $\mathrm{SO}_{4}$ for $6 \mathrm{hr}$ (lanes 2, 4, 6 and 8) or not (lanes 1, 3, 5 and 7). WCEs were analyzed by EMSA with the MREa probe (lanes 1 to 4) or the HSE probe (lanes 5 to 8). The white and black arrowheads indicate the positions of bands retarded by MTF-1 and HSF1, respectively. c, Supershift assay. EMSA was carried out as in b, except that an extra 20-min incubation with the following antibodies was added after the binding reaction: lane 1, no antibody; lane 2, antiHSF1 antibody A (Santa Cruz); lane 3, anti-HSF1 antibody B (Affinity BioReagents); lane 4, anti-HSF2 antibody (Santa Cruz). The black arrows indicate the HSE complexes. The white arrow indicates the slot position.

tains HSE and proximal promoter sequences (Fig. 3a). When pHSP70prCAT was used as a reporter, treatment of the transfectants with $200 \mu \mathrm{M}$ $\mathrm{ZnSO}_{4}$ enhanced CAT expression approximately threefold (Fig. 3b), indicating that transcription from the exogenous $h s p 70$ promoter can be induced probably by Zn-activated endogenous HSF1. Under this assay condition, overexpression of HSF1 markedly enhanced CAT reporter expression (Fig. 3c), but Zn did not increase the expression further. Similarly to the observation in EMSA in Fig. 2, induction of the $h s p 70$ promoter by $\mathrm{Zn}$ was repressed in control cells that were co-transfected with an empty expression vector. With $\mathrm{p} \Delta(-112) \mathrm{CAT}$ reporter, the response to the overexpressed HSF1 was also observed. How- ever, levels of the reporter expression were reduced as compared with the wild type reporter. From these experiments, the proximal region of the $h s p 70$ promoter containing HSE appears to be sufficient for the response to HSF1. Lack of the -270 to -113 sequence resulted in the reduced transcriptional activity, suggesting that there may be other regulatory elements required for the optimal levels of transcription within that region.

\section{DISCUSSION}

Previous reports have suggested that HSE and HSF1 are involved in the metal response of the $h s p 70$ 

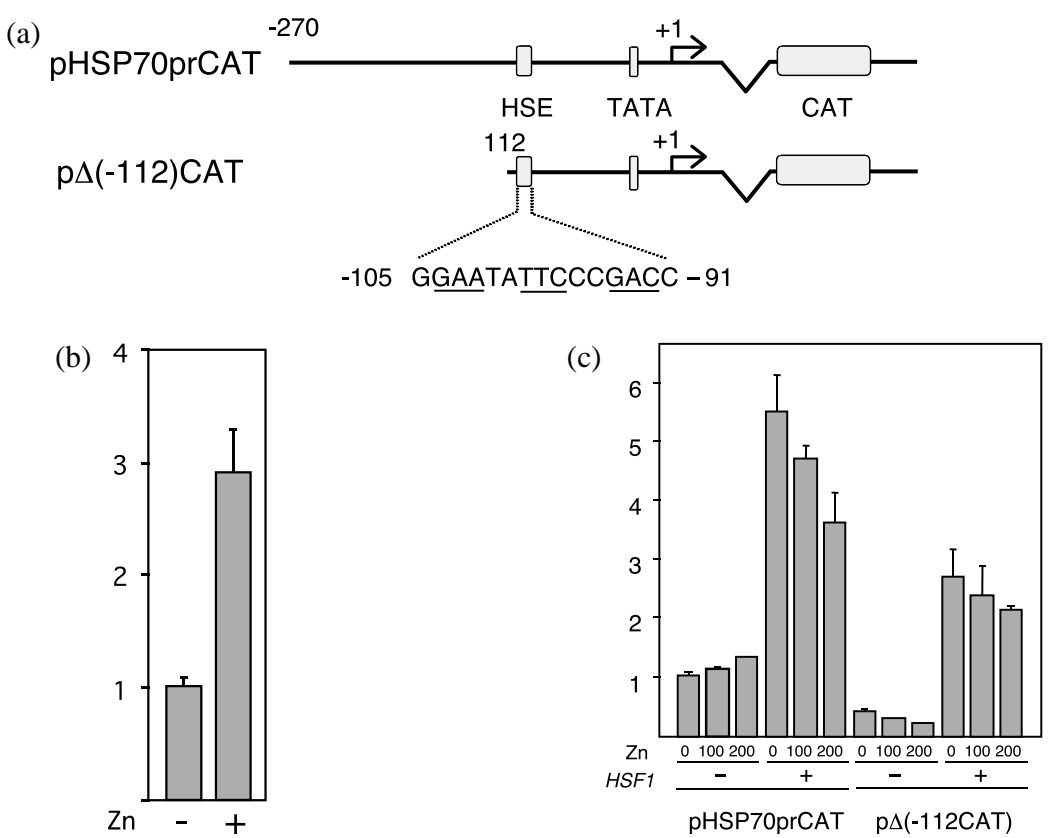

Fig. 3. Effect of HSF1 Overexpression on the $h s p 70$ Promoter Activity

a, Structures of the reporter plasmids. b, Transient transfection assay with pHSP70prCAT as a reporter plasmid. HeLa cells were transfected with pHSP70prCAT and cultured for $46 \mathrm{hr}$. After a 6-hr incubation with or without $200 \mu \mathrm{M} \mathrm{ZnSO}_{4}, \mathrm{CAT}$ reporter expression was determined. Assays were carried out in duplicate, and the averages with standard errors are shown. Relative values are indicated taking that for the control unexposed cells as 1.c, Effects of HSF1 overexpression. HeLa cells were transfected with pHSP70prCAT or p $4(-112)$ CAT. An HSF1 expression vector pCI-HSF1 (+) or an empty vector pCI (-) was co-transfected. After $46 \mathrm{hr}$, the cells were incubated with or without the indicated concentrations of $\mathrm{ZnSO}_{4}$ for $6 \mathrm{hr}$ further. Reporter expression was determined and is indicated as in b. Relative values are indicated taking that for the cells transfected with pHSP70prCAT but not with pCI-HSF1 and not exposed to $\mathrm{Zn}$ as 1 .

gene. $^{5-7)}$ In the present work, we studied Zn-response of the HSE-binding activity in HeLa cells, with regard to both the endogenous and overexpressed recombinant HSF1. The induction of the DNA-binding activity of endogenous HSF1 in a $\mathrm{Zn}$ concentration-dependent manner correlated well to the increase of $h s p 70$ mRNA, ${ }^{7)}$ supporting an idea that HSF1 activation represents the mechanistic background for the transcriptional activation of the $h s p 70$ gene. Our results also showed that the DNA-binding properties of HSF1 and MTF-1 are totally different in $\mathrm{Zn}$ concentration-dependency and inducibility by $\mathrm{Zn}$ in vitro, strongly suggesting that these two transcription factors are activated through distinct mechanisms.

WCE from the cells transfected with an HSF1 expression vector displayed high levels of HSE-binding activity in EMSA. It was unambiguously confirmed that this DNA-binding activity in fact represents HSF1, by supershift analysis. However, Zn exposure did not induce the HSE-binding activity any more, suggesting that the overexpressed HSF1 appears to be constitutively competent for DNA binding. The constitutive transcriptional activity dis- cussed below is likely to reflect this property. Unexpectedly, the metal-induced activation of the endogenous HSF1 was abolished in control cells not transfected with the HSF1 expression vector. This appeared to be caused by the control vector transfected instead, although the mechanism remains uncertain.

In the transient transfection assay with a reporter vector pHSP70prCAT, the overexpression of HSF1 markedly increased the transcription from the $h s p 70$ promoter. However, no Zn response was observed, consistent with the result of EMSA described above. Similar loss of stress response for overexpressed HSF1 has been reported in mouse cells. ${ }^{13)}$ This may reflect that the active form of HSF1 is increased when expressed at high concentrations, or that a transcriptional repressor is titrated out by an excess of HSF1. To examine how this de-repression occurs may provide a clue to the understanding of metal regulation.

Acknowledgements The authors thank Dr. R. I. Morimoto for kindly providing a clone containing the upstream region of the human $h s p 70$ gene, and 
Dr. C. Wu for kindly providing a clone containing the human HSF1 cDNA.

\section{REFERENCES}

1) Koizumi, S. (1997) Analysis of heavy metal-induced gene expression. In Handbook of human toxicology (Massaro E. J., Ed.), CRC Press, Boca Raton, pp. 103-108.

2) Yamada, H. and Koizumi, S. (2002) DNA microarray analysis of human gene expression induced by a nonlethal dose of cadmium. Ind. Health, 40, 159-166.

3) Durnam, D. M. and Palmiter, R. D. (1981) Transcriptional regulation of the mouse metallothioneinI gene by heavy metals. J. Biol. Chem., 256, 57125716.

4) Heuchel, R., Radtke, F., Georgiev, O., Stark, G., Aguet, M. and Schaffner, W. (1994) The transcription factor MTF-1 is essential for basal and heavy metal-induced metallothionein gene expression. EMBO J., 13, 2870-2875.

5) Williams, G. T. and Morimoto, R. I. (1990) Maximal stress-induced transcription from the human HSP70 promoter requires interactions with the basal promoter elements independent of rotational alignment. Mol. Cell. Biol., 10, 3125-3136.

6) Mosser, D. D., Theodorakis, N. G. and Morimoto, R. I. (1988) Coordinate changes in heat shock element-binding activity and $H S P 70$ gene transcription rates in human cells. Mol. Cell. Biol., 8, 4736-4744.

7) Murata, M., Gong, P., Suzuki, K. and Koizumi, S.
(1999) Differential metal response and regulation of human heavy metal-inducible genes. J. Cell. Physiol., 180, 105-113.

8) Koizumi, S., Suzuki, K., Ogra, Y., Gong, P. and Otuska, F. (2000) Roles of zinc fingers and other regions of the transcription factor human MTF-1 in zinc-regulated DNA binding. J. Cell. Physiol., 185, 464-472.

9) Wu, B., Hunt, C. and Morimoto, R. (1985) Structure and expression of the human gene encoding major heat shock protein HSP70. Mol. Cell. Biol., 5, 330-341.

10) Rabindran, S. K., Giorgi, G., Clos, J. and Wu, C. (1991) Molecular cloning and expression of a human heat shock factor, HSF1. Proc. Natl. Acad. Sci. U.S.A., 88, 6906-6910.

11) Koizumi, S., Suzuki, K., Ogra, Y., Yamada, H. and Otsuka, F. (1999) Transcriptional activity and regulatory protein binding of metal-responsive elements of the human metallothionein-IIA gene. Eur. $J$. Biochem., 259, 635-642.

12) Baler, R., Dahl, G. and Voellmy, R. (1993) Activation of human heat shock genes is accompanied by oligomerization, modification, and rapid translocation of heat shock transcription factor HSF1. Mol. Cell. Biol., 13, 2486-2496.

13) Sarge, K. D., Murphy, S. P. and Morimoto, R. I. (1993) Activation of heat shock gene transcription by heat shock factor 1 involves oligomerization, acquisition of DNA-binding activity, and nuclear localization and can occur in the absence of stress. Mol. Cell. Biol., 13, 1392-1407. 